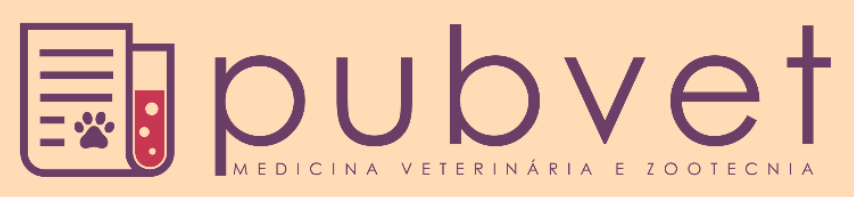

https://doi.org/10.31533/pubvet.v14n3a530.1-7

\title{
A terapêutica do felino diabético: revisão
}

\author{
Isabela Lopes Massite ${ }^{1 *} \bullet$, Danilo Barbosa $\operatorname{Viana}^{2}{ }^{\bullet}$, Jessica Miranda dos Santos $\operatorname{Toyoda}^{30}$, \\ Marcos Ferrante ${ }^{4}$, Melissa Sanches Mongelli5 ${ }^{\bullet}$, Luis David Solis Murgas ${ }^{4}{ }^{\bullet}$, Hugo Shisei Toma $^{4}{ }^{\bullet}$, \\ Fernando Marcos Rubim ${ }^{60}$
}

${ }^{I}$ Discente do curso de Medicina Veterinária - Universidade Estadual de Maringá, campus Umuarama, Brasil

${ }^{2}$ Residente em Clínica de Animais de Companhia - Universidade Estadual de Londrina, Brasil

${ }^{3}$ Médica Veterinária formada pela Universidade Estadual de Maringá, campus Umuarama, Brasil

${ }^{4}$ Docente do curso de Medicina Veterinária - Universidade Federal de Lavras, Brasil

${ }^{5}$ Discente do curdo de Medicina Veterinária - Universidade Federal de Lavras, Brasil

${ }^{6}$ Mestrando no PPGV/DMV - Universidade Federal de Lavras, Brasil

*Autor para correspondência, email: isa_massitel@hotmail.com

Resumo. A Diabetes Mellitus (DM) é uma endocrinopatia muito comum na rotina clínica de pequenos animais. Tal enfermidade, de acordo com sua causa, pode ser classificada em tipo I ou tipo II, sendo a última a mais frequente encontrada nos felinos. Os principais sinais clínicos em gatos podem incluir poliúria, polidpsia, polifagia, anorexia, letargia, pelame seco e sem brilho, além de carência na higiêne dos pelos. O diagnóstico é desafiador e exige uma série de exames para confirmação e início do tratamento. Para se obter sucesso no tratamento e chegar a possível remissão do quadro, deve-se realizar um plano terapêutico individualizado para cada animal, cujo principal objetivo é o controle da glicemia, principalmente através da insulinoterapia e correção da dieta do paciente, sendo este o tema principal desta revisão.

Palavras chave: diabetes, insulina, tratamento

\section{The diabetic feline therapy: review}

\begin{abstract}
Diabetes Mellitus (DM) is a very common endocrinopathy in the clinical routine of small animals. Such disease, according to its cause, can be classified as type I or type II, the latter being the most frequent found in felines. The main clinical signs in cats may include polyuria, polydipsia, polyphagia, anorexia, lethargy, dry and dull fur, and poor hygiene of hair. Diagnosis is challenging and requires a series of tests to confirm and start treatment. To be successful in the treatment and to achieve possible remission of the condition, an individualized therapeutic plan should be made for each animal, whose main objective is the control of blood glucose, especially through insulin therapy and correction of the patient's diet, which is the theme. main part of this review.
\end{abstract}

Keywords: diabetes, insulin, treatment

\section{La terapia felina diabética: revisión}

Resumen. La diabetes mellitus (DM) es una endocrinopatía muy común en la rutina clínica de animales pequeños. Dicha enfermedad, según su causa, puede clasificarse como tipo I o tipo II, siendo esta última la más frecuente en felinos. Los principales signos clínicos en los gatos pueden incluir poliuria, polidipsia, polifagia, anorexia, letargo, pelaje seco y sin brillo, y una mala higiene de los pelos. El diagnóstico es desafiante y requiere una serie de pruebas para confirmar y comenzar el tratamiento. Para tener éxito en el tratamiento y lograr la posible remisión de la afección, se debe elaborar un plan terapéutico 
individualizado para cada animal, cuyo objetivo principal es el control de la glucosa en sangre, especialmente a través de la terapia con insulina y la corrección de la dieta del paciente, que es el tema. parte principal de esta revisión.

Palabras clave: diabetes, insulina, tratamiento

\section{Introdução}

A Diabetes Mellitus (DM) pode ser descrita como um distúrbio pancreático endócrino que leva a uma deficiência parcial ou total de insulina no organismo do animal, acarretando em um estado de hiperglicemia persistente (Rocha, 2018). Tal enfermidade é classificada em tipo I (DM insulinodependente) e tipo II (DM não dependente de insulina), a primeira é caracterizada pela destruição das células pancreáticas com perda progressiva e completa da produção insulínica, sendo rara em gatos. $\mathrm{O}$ tipo II, responsável por $80 \%$ dos casos em felinos, é caracterizada por uma resistência insulínica e/ou por disfunção das células beta pancreáticas (Nelson \& Couto, 2015).

As causas mais comuns em gatos são resistência insulínica, amiloidose nas Ilhotas Pancreáticas e Pancreatite Linfoplasmocítica Crônica. Além disso, alguns fatores de riscos também são intimamente relacionados, como obesidade, acromegalia, doença renal, hiperadrenocorticismo, infeccções sistêmicas, pancreatite e gravidez/estro (Behrend et al., 2018). Independentemente da etiologia subjacente, os sinais clínicos clássicos de poliúria (PU), polidipsia (DP), polifagia (PP) e perda de peso resultam de hiperglicemia prolongada e glicosúria.

Os felinos diagnosticados geralmente possuem média de idade de 10 anos (Nelson \& Couto, 2015), com maior prevalência em machos castrados e obesos (Crivellentin \& Borin-Crivelletin, 2015). Fêmeas também podem ser acometidas, principalmente aquelas não castradas, devido à resistência insulínica induzida pela progesterona durante o estro (Sparkes et al., 2015).

Em relação a terapia pode-se dizer que o tratamento é complicado, pois abrange desde a compreensão das evidências científicas atuais e bom julgamento clínico.O tratamento inicial depende das alterações clínicas e físicas do animal. A princípio, é feito a administração de insulinoterapia, correção da dieta do paciente e principalmente o controle da glicemia (Nelson \& Couto, 2015).

\section{Fisiopatologia}

A insulina é um homônio secretado pelas células $\beta$ nas ilhotas de Langerhand do pâncreas. Diversos mecanimos permitem a liberação do insulina (๑), como os grânulos de armazenamento nas células $\beta(\boldsymbol{O})$, porém o principal é o aumento de glicose plasmática (hiperglicemia).

Em um animal sadio, para a maioria dos órgãos, a insulina precisa se ligar a receptores de insulina na periferia de células, para possibilitar a entrada de glicose vinda da corrente sanguínea para o interior da célula. Quando a insulina se liga ao receptor, são ativados mecanismos intracelulares, os quais resultam em transportadores de glicose movimentando-se na direção da membrana celular. Ao menos 12 proteínas transportadoras de glicose (GLUT: glucose transporter proteins) foram descritas e o GLUT4 é o responsável pela captação de glicose mediada por insulina. As vesículas de GLUT4 ancoram na membrana celular, realizando assim, a fusão entre ambas e tornando-se possível a difusão intracelular de glicose. Este é um processo complexo, mediado por pelo menos três genes em mamíferos (Feldman, 1997; Jericó et al., 2015; Little, 2016).

Segundo Jericó et al. (2015), independentemente do motivo pelo qual a insulina não consegue exercer adequadamente os seus efeitos biológicos, irá ocorrer à hiperglicemia crônica e o efeito de glicotoxicidade. Esse efeito contribui para a redução da secreção da insulina, determinando a resistência à insulina em tecidos periféricos e interferindo nos mecanismos de transporte da glicose. Os autores Norsworthy et al. (2004) afirmam que provavelmente o resultado da falha das células $\beta$ é a causa subjacente da diabetes, combinada com a supressão de secreção de insulina por toxicidade da glicose, como observado na figura 1. Esta supressão, é inicialmente funcional e reversível, no entanto, ao longo de semanas e meses, causa danos irreversíveis às células $\beta$ e perda das mesmas. Este fato explica em grande parte porque os gatos com diabetes mal controlada por mais de 6 meses, reduziram significativamente a probabilidade de remissão, mesmo após o bom controle glicêmico. 


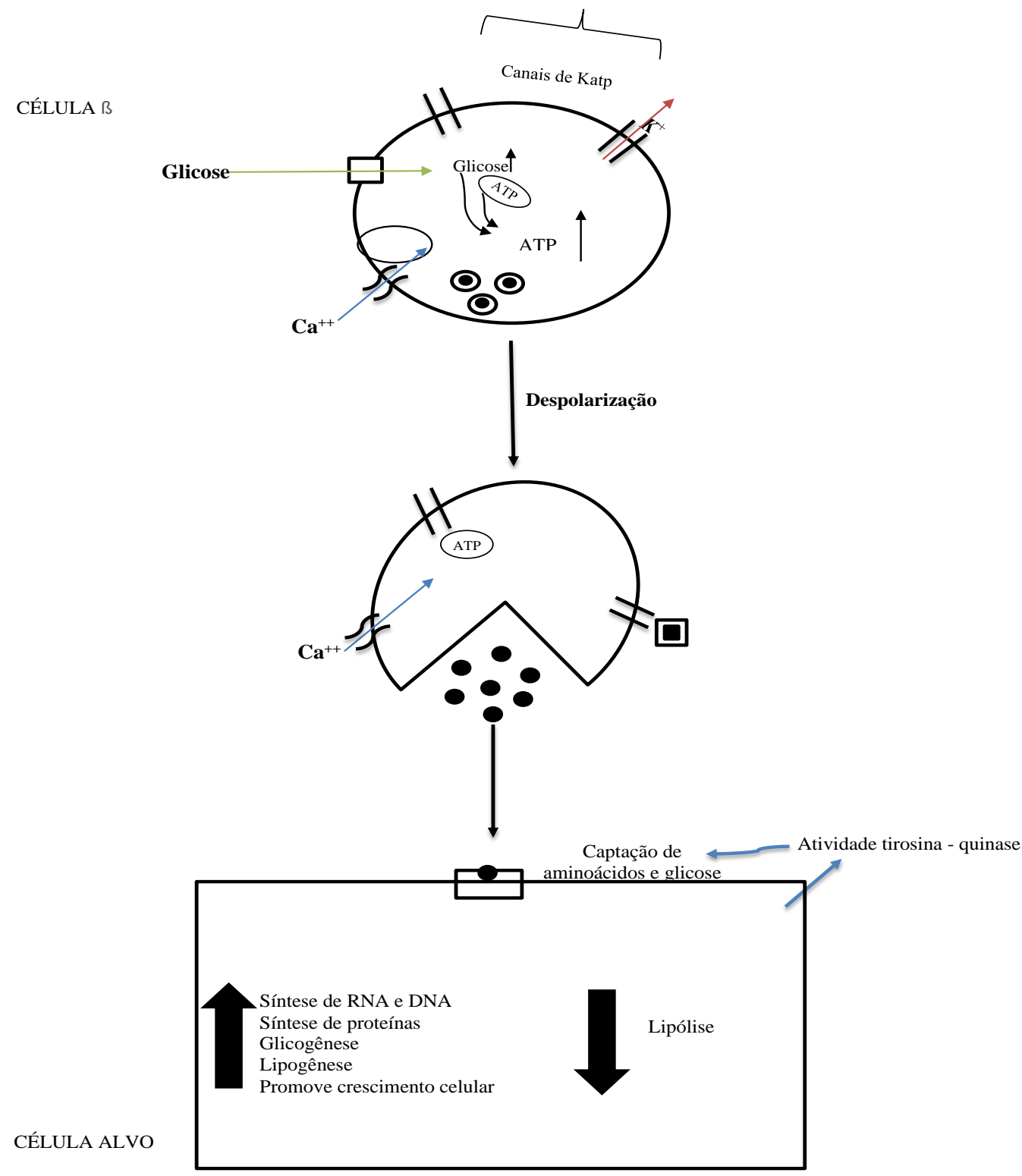

Figura 1. Fisiopatologia da Diabetes Mellitus.

\section{Diagnóstico}

Para o diagnóstico, primeiramente procede-se a realização de minuciosa anamnese seguida do completo exame físico do paciente, associado aos exames laboratoriais. Hemograma completo, exames bioquímicos hepáticos e renais, dosagem de eletrólitos e frutosamina, hemogasometria, urinálise e cultura da urina, relação proteína: creatinina urinária e pressão arterial são necessários para dar base ao diagnóstico da DM e excluir outras doenças concomitantes (Behrend et al., 2018; Nelson \& Couto, 2015). Geralmente há uma suspeita muito forte quando ocorrem hiperglicemia e glicosúria persistentes, além de consistentes sinais clínicos (Sparkes et al., 2015). Em felinos, a mensuração da frutosamina é extremamente útil para exclusão da hiperglicemia por estresse, muito comum nessa espécie (Crivellentin \& Borin-Crivelletin, 2015).

$\mathrm{Na}$ avaliação laboratorial tem-se o achado típico para DM, o qual incluí hiperglicemmia, glicosúria e leucograma de estresse, além do aumento de colesterol, triglicerídeos e ALP. No exame de urina, observa-se presença de glicose, protéinas, cetonas e bactérias. Todavia, em casos que há ausências de sinais clínicos consistentes, pode-se indentificar o BG no exame de sangue. Em tais casos, se a hiperglicemia por estresse for descartada, o paciente pode ser classificado como risco para o desenvolvimento da DM. A glicosúrio normalmente desenvolve-se quando a concentração de BG excede 230-300 mg/dL (Crivellentin \& Borin-Crivelletin, 2015). 
É importante salientar que as concentrações de glicose no sangue podem variar entre os níveis de referência, quando ocorre tal situação deve-se considerar outros motivos para tal aumento, como, estresse, administração de corticoides, hiperadrenocoirticismo, obesidade e etc.

Em relação aos sinais clínicos (UP, DP,PP e perda de peso), vão estar presentes quando houver hiperglicemia e glicosúria persistente. No entando, em estágios iniciais da doneça não é comum a observação sinais clínicos.

Caso o quadro clínico persista sem tratamento adequado, ao exame físico pode demostrar desistração, pelo opaco, dor abdominal com pancreatite concomitante. Gatos com hiperglicemia presente ao longo do tempo pode desenvolver neuropatia periférica e cetose.

\section{Tratamento}

No geral, o tratamento baseia-se na aplicação de insulina e modificação da dieta, com o objeito de controlar o BG baixo por 24 horas, o qual melhora os sinais clínicos e evita complicações comcomitantes. Para o manejo bem sucedido da DM, deve-se obter a percepção do proprietária em forncer boa qualidade de vida e tratamento adequado para o animal. Preditores de remissão diabética em gatos possuem o objetivo de alcançar um controle glicêmico dentro de seis meses, com o uso de monitoramento domiciliar intensivo, insulina glargina ou detemir e baixo teor de carboidratos na dieta.

\section{Insulinoterapia}

O tratamento inicial realizado é a insulinoterapia com glargina ou protamina-zinco insulina (1-2 unidades/gato/12horas). Pode-se ou não monitoriar a BG em decorrer do tratamento, tal opção é critério do veteriário. Deve-se diminuir a dose de insulina em $50 \%$ caso a glicemia estiver em $150 \mathrm{mg} / \mathrm{dL}$. Sugere-se reaavaliar o animal em 7 a 14 dias.

As insulinas podem ser de ação lenta, intermediária ou rápida. Antigamente, eram utilizadas as insulinas de ação intermediária ou rápida, causando picos hipoglicemicos em felinos, causando as hiperglicemias de rebote (efeito somogyi) e descontrole da doença (Rand \& Marshall, 2005). Como o pico de duração da insulina era rápido e o controle glicêmico ineficaz, devido a alimentação do felino ser constante durante o dia em poucas quantidades, não ocorria o efeito desejado da insulina.

Os produtos compostos de insulina são diversos: 1. Lente (suspensão de insulina/zinco) - tem ação intermediária (12 horas); 2. Glargina - possui ação mais longo, sendo considerada a insulina de eleição para gatos com DM; 3. PZI (insulina recombinate humana de zinco protamina U-40), possui ação prolongada ( 8 - 24horas), mais utilizada em cães; 4. Determir (recombinate humano U-100), com ação prolongada é analógica a insulina humana, é considerada semelhante à glargina.

Para o tratamento de felinos diabéticos prioriza-se o uso de insulina de longa duração - Glargina e Detemir. A insulina Glargina não apresenta picos de ação, sendo solúvel em $\mathrm{pH}$ 4,0, possuindo via de administração injetável (Gilor \& Graves, 2010). Quando administrada, é precipitada devido seu pH neutro do tecido subcutâneo, gerando microcristais liberados e absorvidos constantemente por um longo período, possibilitando assim, menores quadros de hipoglicemia (Gilor \& Graves, 2010). Tal medicamento possui ação de até $24 \mathrm{~h}$ possuindo seu pico de ação na terceira hora após administração (Bloom \& Rand, 2014). É preconizada a administração da insulina duas vezes ao dia (BID) por diminuir os riscos de picos hipoglicêmicos, apesar de haver felinos que não possuem tal necessidade (Rand \& Marshall, 2005). A dose é feita de acordo com o nível glicêmico do animal e o seu peso, caso a glicemia estiver $\geq 360 \mathrm{mg} / \mathrm{dL}$, realiza-se $0,5 \mathrm{U} / \mathrm{Kg}$ duas vezes ao dia de insulina, caso estiver $\leq 360 \mathrm{mg} / \mathrm{dL}$, diminui-se a dose em 50\%, ou seja, administra-se $0,25 \mathrm{U} / \mathrm{kg}$ duas vezes ao dia, como observado na figura 2. É importante lembrar que a glargina não pode ser diluída devido sua absorção lenta, baixo pH e interação com gordura subcutânea.

A insulina Detemir possui a dose igual a Glargina, sendo demonstrado na figura 2. Possui efeito longo e diminuindo o ganho de peso em pacientes que fazem seu uso. Em um estudo recente feito por Hoelmkjaer et al. (2015), a insulina Detemir obteve índice de remissão de 67\% nos felinos diabéticos com tempo de diagnóstico variável. Naqueles com diagnóstico menor que dois meses, a remissão foi de $91 \%$. 
De acordo com Gottlieb \& Rand (2013), há relatos que o uso de corticosteróide nos 6 meses antes do diagnóstico da diabetes foi significativamente associado a maiores taxas de remissão e, em um estudo citado pelos autores, todos os gatos com uso prévio de corticosteróide alcançaram remissão. Uma explicação para isso é que os gatos com diabetes associada à administração de corticosteróides apresentaram um aparecimento de sinais clínicos e puderam ser diagnosticado mais rapidamente. A terapia precoce reduz a duração da hiperglicemia, por isso, reduz os danos as células $\beta$. Outra consideração é que o diabetes mellitus induzido por drogas é considerado ser um tipo diferente de diabetes do tipo 2, portanto o processo da patologia subjacente pode ser reversível de forma mais fácil.

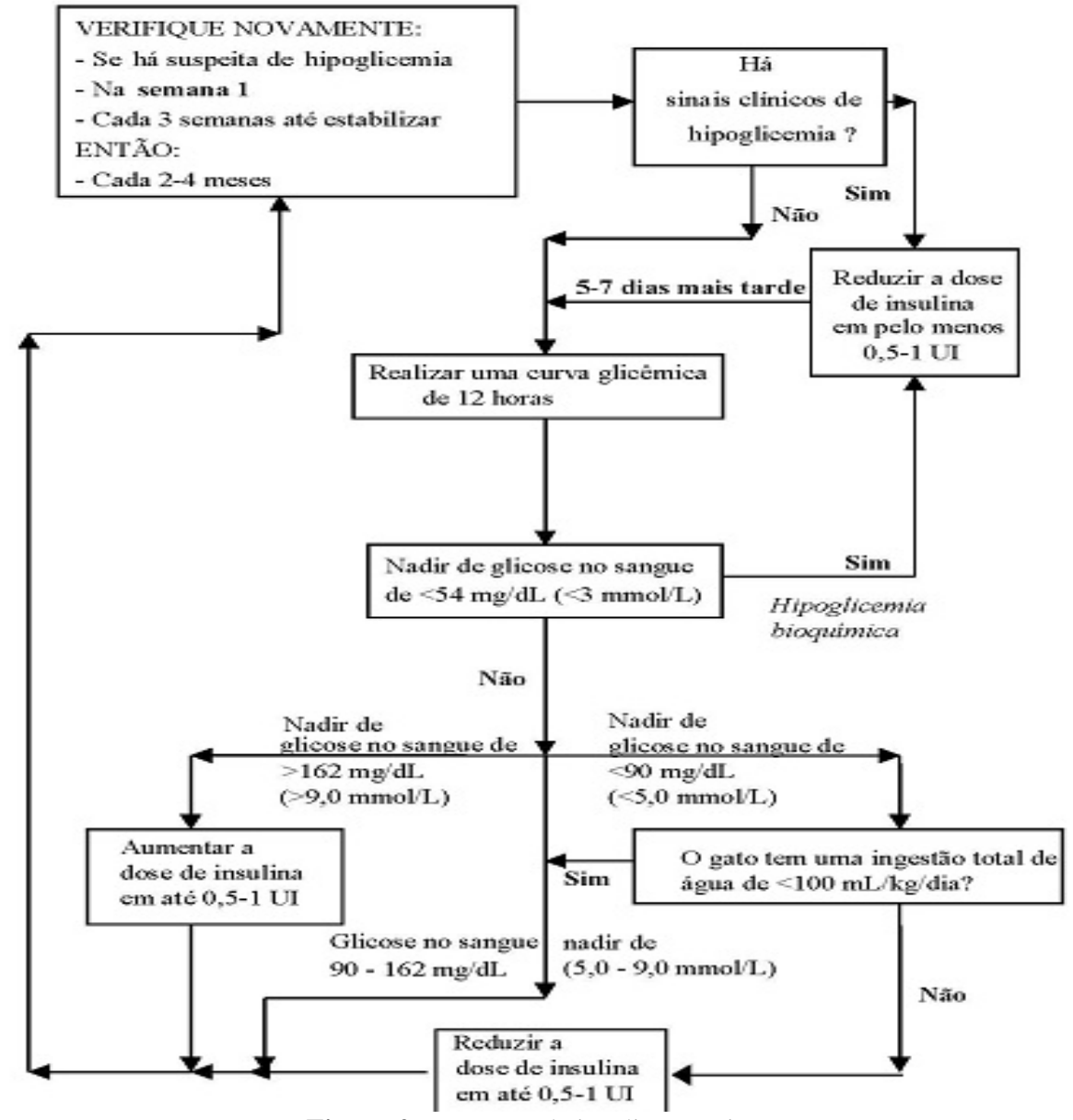

Figura 2. Dosagem da insulinoterapia.

De modo geral, em relação aos dosegens de insulina devem ser baseadas no peso corporal do paciente. Na maioria dos casos, recomenda-se incialmente $1 \mathrm{U}$ de Glargina a cada 12 horas. Mesmo em um gato muito grande, a dosagem inicial não debe exceder $2 \mathrm{U}$ a cada 12 horas. Em geral, utiliza-se o equilíbrio de $0,5 \mathrm{U} / \mathrm{kg}$ a cada 12 horas.

\section{Agentes terapêuticos não insulínicos}

Em alguns casos específicos pode-se utilzar fármacos não associados a insulina como observado na Tabela 1.

1. Sulfonilureias (Glipizida): promovem secreção de insulina do pâncreas. Pesquisas demonstram que a utlização de glipizida via oral obteve-se benefícios em $40 \%$ dos gatos tratados. Podem ocorrer efeitos adversos, como colestase, hipoglicemia e vômito. A dose incial baseia-se em $2,5 \mathrm{mg} / \mathrm{gato}$ via oral a cada 12 horas, concomitante com o manejo da dieta. Caso não for observado resposta ao tratamento deve-se instituir a insulinoterapia.

2. Inibidores de $\alpha$-glucosidades (acarbose): inibem a absorção intestinal de glicose e diminuem a hiperglicemia pós-alimentação. Terapia utilizada juntamente com uma dieta pobre em carboidratos. Pode estar presente como efeito colateral diarréia. 
3. Incretinas com GLP-1 (peptídeo semelhante ao glucagon 1) - São hormônios que podem ser utilizados juntamente com Glargina. São responsáveis por aumentar a secreção de insulina e proteção às élulas $\beta$ da oxidação.

Tabela 1. Classes de agentes terapêuticos não insulínicos

\begin{tabular}{lclc}
\hline Classe & Exemplo & Mecanismo de ação & $\begin{array}{l}\text { Concomitante à } \\
\text { insulinoterapia }\end{array}$ \\
\hline Sulfonilureias & Glipizida & Secreção de insulina do pâncreas & Não \\
\hline Inibidores de $\alpha$-glucosidades & Acarbose & $\begin{array}{l}\text { Inibição da absorção intestinal de glicose e } \\
\text { diminuição da hiperglicemia pós-alimentação }\end{array}$ & Sim \\
\hline Incretinas com GLP-1 & $\begin{array}{c}\text { Peptídeo semelhante } \\
\text { ao glucagon 1 }\end{array}$ & $\begin{array}{l}\text { Aumento da secreção de insulina e proteção às } \\
\text { células B da oxidação. }\end{array}$ & Sim \\
\hline
\end{tabular}

\section{Dieta}

Tem como objetivo a perda de peso em animais obesos gerando diminuição na hiperglicemia pósprandial para o aumento da chance de remissão pós diagnóstico. É prescrito uma dieta hipocalórica com baixos índices de carboidratos, gorduras e com altas concentrações de proteínas e fibras para obter saciedade. Atualmente há ações já formuladas no mercado que oferecem a terapêutica necessária para o felino (Zoran \& Rand, 2013).

A meta de perda de peso em gatos obesos é uma redução 0,5 a $2 \%$ por semana. Gatos diabéticos devem receber uma dieta rica em proteínas, para maximizar a taxa metabólica e evitar o risco de lipodose hepática durante a perda de peso.

\section{Hipoglicemiantes orais}

São utilizados apenas com proprietários que possuem aversão em realizar o tratamento com insulina devido a sua administração ser intravenosa. Estão em desuso devido sua ineficácia durante o tratamento. A Glipizida é o único fármaco que tem evidências comprovadas no controle glicêmico, no entanto é ineficaz na metade dos casos e induz efeitos indesejáveis (Bloom \& Rand, 2014; Rand, 2013; Reusch, $\underline{2010)}$.

\section{Monitoramento}

O objetivo pricipal do monitoramento é controlar os sinais clínicos de DM e previnir a hipoglicemia, ou seja, manter a BG abaixo do seu limiar $(250-300 \mathrm{mg} / \mathrm{dL})$. As opções de monitoramento incluem a mensuração da BG, UG, da frutosamina e avaliando os sinais clínicos e o peso corporal.

É importante salientar que é necessário que a monitoração seja acompanhada por sinais clínicos, caso o animais não demostre nenhum sinal já citado anteriormente é que ele está estável. Gatos devem ser monitorados com maior frequência e atenção. A mensuração da $B G$ é a única maniera que identifica hipoglicemia, porém não é um parâmetro confiável utilizado sozinho. A monitoração mais confiável é a verificação de BGCs, no entando ainda é pouco utilizada na medicina veterinária.

\section{Conclusão}

Pode-se concluir a partir deste trabalho que o melhor plano terapêutico para o felino diabético é a remissão de seu estado. Fatores como o diagnóstico precoce, a utilização da insulina de longa ação e dose correta, dieta pobre em carboidratos, controle glicêmico, observação e acompanhamento veterinário são decisivos para um resultado positivo.

Além disso, nota-se que para o sucesso da gestão da DM o tutor deve estar comprometido no tratamento. Com isso, pode-se estabelecer uma educação proativa ao proprietário do animal, com instruções sobre a insulinoterapia, de agentes não insulínicos e da dieta.

\section{Referências bibiliográficas}

Behrend, E., Holford, A., Lathan, P., Rucinsky, R., \& Schulman, R. (2018). 2018 AAHA diabetes 
management guidelines for dogs and cats. Journal of the American Animal Hospital Association, $54(1), 1-21$.

Bloom, C. A., \& Rand, J. (2014). Feline diabetes mellitus: clinical use of long-acting glargine and detemir. Journal of Feline Mdicine and Surgery, 16(3), 205-215.

Crivellentin, L. Z., \& Borin-Crivelletin, S. (2015). Casos de rotina em medicina veterinária de pequenos animais. In MedVet.

Feldman, E. C. (1997). Tratado de medicina interna veterinária. In Moléstias do cão e do gato (Vol. 3).

Gilor, C., \& Graves, T. K. (2010). Synthetic insulin analogs and their use in dogs and cats. Veterinary Clinics: Small Animal Practice, 40(2), 297-307.

Gottlieb, S., \& Rand, J. S. (2013). Remission in cats: including predictors and risk factors. The Veterinary Clinics of North America. Small Animal Practice, 43(2), 245-249.

Hoelmkjaer, K. M., Spodsberg, E.-M. H., \& Bjornvad, C. R. (2015). Insulin detemir treatment in diabetic cats in a practice setting. Journal of Feline Mdicine and Surgery, 17(2), 144-151.

Jericó, M. M., Kogika, M. M., \& Andrade Neto, J. P. (2015). Tratado de medicina interna de cães e gatos. Guanabara Koogan.

Little, S. E. (2016). O gato: medicina interna. Editora Roca.

Nelson, R. W., \& Couto, C. G. (2015). Medicina interna de pequenos animais (Issue 1). Elsevier Editora.

Norsworthy, G. D., Crystal, M. A., Grace, S. F., \& Tilley, L. P. (2004). O paciente felino. São Paulo: Roca, 3, 300 .

Rand, J. S. (2013). Pathogenesis of feline diabetes. Veterinary Clinics: Small Animal Practice, 43(2), 221-231.

Rand, J. S., \& Marshall, R. D. (2005). Diabetes mellitus in cats. Veterinary Clinics: Small Animal Practice, 35(1), 211-224.

Reusch, C. (2010). Feline diabetes. In S. J. Ettinger \& E. C. Feldman (Eds.), Textbook of veterinary internal medicine (pp. 1796-1816). Saunders.

Rocha, M. B. (2018). Fatores de risco para diabetes mellitus felina: revisão de literatura. Universidade Federal do Rio Grande do Sul.

Sparkes, A. H., Cannon, M., Church, D., Fleeman, L., Harvey, A., Hoenig, M., Peterson, M. E., Reusch, C. E., Taylor, S., \& Rosenberg, D. (2015). ISFM consensus guidelines on the practical management of diabetes mellitus in cats. Journal of Feline Mdicine and Surgery, 17(3), 235-250.

Zoran, D. L., \& Rand, J. S. (2013). The role of diet in the prevention and management of feline diabetes. Veterinary Clinics: Small Animal Practice, 43(2), 233-243.

Recebido: 14 de janeiro, 2020.

Aprovado: 10 de fevereiro, 2020.

Publicado: 22 de abril, 2020.

Licenciamento: Este artigo é publicado na modalidade Acesso Aberto sob a licença Creative Commons Atribuição 4.0 (CC-BY 4.0), a qual permite uso irrestrito, distribuição, reprodução em qualquer meio, desde que o autor e a fonte sejam devidamente creditados. 